\title{
Ballistic electron transport in stubbed quantum waveguides: experiment and theory
}

\author{
P. Debray ${ }^{1}$, O. E. Raichevt, P. Vasilopoulos \\ R. Perrin ${ }^{1}$, and W. C. Mitchell ${ }^{1}$ \\ 1 Air Force Research Laboratory, AFRL/MLPO, Wright-Patterson AFB, Ohio, USA \\ 甘Service de Physique de l' Etat Condense, CEA de Saclay, 91191 Gif-sur-Yvette, France \\ オInstitute of Semiconductor Physics, NAS, 45 Prospekt Nauki, 252650 Kiev, Ukraine \\ 日Concordia University, Department of Physics, Montréal, Québec, Canada, H3G 1M8 \\ ${ }^{2}$ Department of Physics, University of Glasgow, Glasgow, G12 8QQ, UK
}

\begin{abstract}
We present results of experimental and theoretical investigations of electron transport through stub-shaped waveguides or electron stub tuners (ESTs) in the ballistic regime. Measurements of the conductance $G$ as a function of voltages, applied to different gates $V_{i}(i=$ bottom, top, and side) of the device, show oscillations in the region of the first quantized plateau which we attribute to reflection resonances. The oscillations are rather regular and almost periodic when the height $h$ of the EST cavity is small compared to its width. When $h$ is increased, the oscillations become less regular and broad depressions in $G$ appear. A theoretical analysis, which accounts for the electrostatic potential formed by the gates in the cavity region, and a numerical computation of the transmission probabilities successfully explains the experimental observations. An important finding for real devices, defined by surface Schottky gates, is that the resonance minima result from size quantization along the transport direction of the EST.
\end{abstract}

PACS: 73.61.-r, 85.30.Vw

(November 10, 2018)

\section{INTRODUCTION}

Submicron-size T-shaped electron waveguides, defined electrostatically in a twodimensional electron gas (2DEG) by Schottky gates, are very promising devices for potential applications in microelectronics since their conductance $G$ is determined, in the ballistic regime, by quantum interference effects and can be changed by applying voltages to the gates [1]. Such devices, commonly known as Electron Stub Tuners (ESTs), also open the way for studying resonant states of ballistic quantum dots in both the weakly coupled tunneling and in the transmissive open regime [2]. The size of an EST can be controlled by gate voltages, cf. Fig. 1. For a theoretical analysis, an EST can be considered as a rectangular quantum dot connected to 2DEG reservoirs through two oppositely placed Quantum Point Contacts (QPC). When the electron phase-coherence length exceeds the dimensions of the EST, transport through the device is ballistic. A number of theoretical papers [3], [4] have been published on the ballistic transport characteristics of ESTs in the open regime of the QPCs. These works predict an oscillatory dependence of $G$ as a function of geometrical size parameters of the device or of the Fermi energy $E_{F}$. A minimum in $G$, or a reflection 
resonance, is said to occur due to resonant reflection of electron waves by quasibound states of the stub cavity (SC) formed by the gates, the quasibound state itself resulting because of the quantization of electron momentum associated with the small device size. Experimentally, it is possible to probe these resonance states through measurements of $G$ at low temperatures and has been reported [4] only very recently. However, so far experimenters have failed to observe well-defined, regular oscillations in $G$ with mimima corresponding to excitations of the quasibound states as predicted theoretically. Most of the devices so far used in experiments were geometrically defined by only two gates, which do not allow an adequate independent control of the width of the QPCs and the shape of the SC. This possibly explains the failure to observe experimentally a well-defined, regular pattern of minima in $G$.

In this paper we present experimental and theoretical results for the four-gate EST. A preliminary account of some of them has appeared recently [5]. We report the experimental observation of a clear and pronounced oscillatory dependence of the ballistic $G$ on the size of the $\mathrm{SC}$ as the latter is changed by voltage-biasing the gates. Such oscillations occur on the first conductance plateau of the QPCs. We also present theoretical results for $G$ obtained from a numerical solution of Schröedinger's equation for a two-dimensional (2D) hard-wall electron waveguide with a shape close to the one resulting from the biasing of the Schottky gates. Because of this choice, we believe our results are closer to reality than those reported in previous theoretical work based on a rectangular approximation for the SC shape. A rectangular SC shape is unrealistic since, though the lithographically defined device shape is rectangular, the shape of the SC changes as the gates are biased [6]. Moreover, except for a few papers [7], the lengths of the QPCs have been considered as infinite; this is a very rough approximation for real devices and we avoid it in our computations. Comparison of the experimentally observed features of the ballistic $G$ to those obtained numerically enables us to determine the physical origin of these features and helps us understand what the shape of the SC is and how it can be modified by applied gate voltages.

The paper is organized as follows. In Sec. II we give a brief description of the device fabrication and measurement techniques and then present results of conductance measurements as function of gate voltages. Section III outlines the theoretical model and of the calculations presents numerical results. Finally, an interpretation of the experimental results based on the theoretical analysis of Sec. III. and conclusions follow in Sec. IV.

\section{EXPERIMENTAL ASPECTS}

\section{A. Device fabrication and measurement techniques}

The ESTs used in this study were fabricated from modulation doped AlGaAs/GaAs heterostructure wafers grown by MBE and having a two-dimensional electron gas (2DEG) at a depth of $80 \mathrm{~nm}$ below the surface. The carrier concentration $n_{2 D}$ of the 2DEG was $2.4 \times 10^{15} \mathrm{~m}^{-2}$ with a mobility $\mu$ of $100 \mathrm{~m}^{2} / \mathrm{V} \mathrm{s}$ at $4.2 \mathrm{~K}$. These values of $n_{2 D}$ and $\mu$ give a 2DEG Fermi energy $E_{F}=8 \mathrm{meV}$. The ESTs were defined by four Schottky gates S1, T, S2, and B patterned by electron beam lithography on the surface of the wafer. Figure 1 gives a schematic drawing of an EST device while Fig. 2 shows a scanning electron micrograph of a fabricated EST sample. Lighter areas are the Schottky metal gates on the 
wafer surface. The central part of the EST, where the SC is located, forms a lithographically rectangular planar quantum dot of length 0.55 and width $0.25 \mu \mathrm{m}$. The lithographic lengths of the QPCs is $0.1 \mu \mathrm{m}$. The samples were clamped to the mixing chamber of a dilution refrigerator. Considerable care was taken to ensure good thermal contact to the sample. The two-terminal conductance $\mathrm{G}$ of the devices was measured at $90 \mathrm{mK}$ as function of a gate voltage, while the other gates were biased at fixed voltages. Standard low-bias, lowfrequency, lock-in techniques were used to measure $\mathrm{G}$, which was corrected for a low series resistance due to the 2DEG reservoirs. A source-drain rms excitation of $10 \mu \mathrm{V}$ was typically used to drive a current along the length (x-direction) of the QPCs.

Since the four gates are independent, it was possible to characterize the QPCs of the EST device independently by biasing the appropriate gates while grounding the rest of them. High-quality, well-defined conductance quantization staircase was observed for both QPCs. The gates of the QPCs were negatively biased to assure fundamental-mode transport through them. The conductance $\mathrm{G}$ of the device was then measured as function of the size of the SC by sweeping the voltage $V_{T}$ of the top gate, or $V_{B}$ of the bottom gate, or $V_{S}$ of the side gates, while the other gates were biased at fixed negative voltages. The sweeping gate voltage was changed until the device was completely pinched off, allowing measurements in both the single-mode open and the tunneling regime of electron transport. Measurements were made on a few EST devices. All of them gave nearly identical and reproducible results differing only in the pinch-off voltages.

\section{B. Experimental results}

Figures 3 to 7 show the experimental results. Very well-defined oscillations in the ballistic conductance $\mathrm{G}$ are observed as a function of a sweeping gate voltage, which changes the size of the SC, while the other gates are biased at fixed voltages. These oscillations exhibit several features which are found to be generic to the EST devices studied. All results shown correspond to transport in the fundamental mode through the QPCs until they are pinched off.

Figure 3 shows the oscillations in $\mathrm{G}$ observed when the bias voltage $V_{T}$ of the top gate is swept, while the other gates are kept at fixed voltages. The solid curve is obtained when the bottom and the two side gates are biased, respectively, at $V_{B}=-765 \mathrm{mV}, V_{S 1}=-860$ $\mathrm{mV}$, and $V_{S 2}=-964 \mathrm{mV}$. As $V_{T}$ is made more negative the size of the SC shrinks. This also adds to the depletion due to the side gates and narrows the QPCs until they are pinched off when the device conductance drops to zero. The oscillations in $G$ are found to occur in two distinct regimes of the sweeping gate voltage, one for which $G<e^{2} / h$ and the other for which $G>e^{2} / h$. The one with $G<e^{2} / h$ is the tunneling regime when $E_{F}$ is below the bottom of the lowest conduction subband of the QPCs, which now form energy barriers through which electrons can tunnel. The oscillations of $\mathrm{G}$ in this regime are found to be periodic, quite sharp, and well resolved. The regime for which $G>e^{2} / h$ may be called the open regime. This happens when $E_{F}$ is above the bottom of the lowest subband of the QPCs such that transport is in the fundamental mode. The $G$ oscillations in this regime are located on the first quantized conductance plateau of the QPCs. Though quite robust, clear, and nearly periodic, they are relatively broad and show a certain degree of overlapping with the resulting convolution effect. The peak values are less than the expected quantized value 
of $2 e^{2} / h$ and, as will be seen later, result from backscattering at the QPC entrance and/or from boundary roughness at the QPC walls. The dotted $G$ curve of Fig. 3 is obtained for $V_{B}=-780 \mathrm{mV}$ and differs from the solid one in two important respects. First, the average conductance is substantially lower. Second, the oscillations in $G$ in the open regime become irregular due to the appearance of broad troughs or depressions in the conductance.

The size of the central ballistic cavity of the device can also be altered by varying the bias voltages of the side gates while keeping those of the top and bottom gates at fixed values. Figure 4 shows the variation in $G$ as function of the side gate bias voltage $V_{S}$ $\left(V_{S}=V_{S 1}=V_{S 2}+104 \mathrm{mV}\right)$. The solid curve was generated with $V_{T}=-1400 \mathrm{mV}$ and $V_{B}=-755 \mathrm{mV}$, while the dotted curve was obtained for $V_{T}=-800 \mathrm{mV}$ and $V_{B}=-790$ $\mathrm{mV}$. Results obtained by sweeping $V_{B}$ are illustrated in Fig. 5. For these measurements the side gates were biased as follows: $V_{S 1}=-860$ and $V_{S 2}=-964 \mathrm{mV}$. The solid and dotted curves correspond, respectively, to $V_{T}=-1085$ and $-635 \mathrm{mV}$. Comparing the results of Figs. 4 and 5 to those of Fig. 3, we notice that, except for the device pinch-off voltages and the oscillation periods, the features of the oscillations in $G$ are similar. The solid curves show the same characteristics, as do the dotted ones, though the features are different for the two sets. This is not surprising since in all cases we are changing the size of the central cavity. An interesting question, however, is what causes the difference in the characteristic features of the $G$ oscillations observed on the first conductance plateau for the solid and the dotted curves. If we look more closely and compare the constant gate bias voltages used for generating the two sets of $G$ curves, an empirical consistency emerges. The voltages used for the dotted curves are such as to result in a $\mathrm{SC}$ which is long compared to that for the corresponding solid curves. As an example, the oscillatory $G$ of the dotted curve in Fig. 4 is obtained for a $V_{T}=-800 \mathrm{mV}$, while for the solid curve $V_{T}$ is equal to $-1400 \mathrm{mV}$. A more negative top gate voltage certainly makes the SC shorter. These observations lead us to conclude that a lower average value of $G$ and broad depressions in it occur when the stub cavity is long. We call the oscillatory $G$ pattern with regular minima observed for the solid curves a "regular" pattern and that for the dotted curves an "irregular" pattern.

In order to better understand the origin of the observed oscillations in $G$ and to distinguish between the peaks in the tunneling and the open regime, we have studied the dependence of the regular $G$ pattern on temperature, drain-source excitation voltage, and a magnetic field applied perpendicular to the plane of the 2DEG. Figure 6 shows the temperature dependence of a regular $G$ pattern obtained by sweeping the top gate voltage $V_{T}$ with $V_{B}=-765 \mathrm{mV}, V_{S 1}=-860 \mathrm{mV}$, and $V_{S 2}=-964 \mathrm{mV}$. As the temperature is increased, all peaks in both the tunneling and the open regime broaden, and eventually they are washed out. At $4.2 \mathrm{~K}$, the oscillatory $G$ pattern has disappeared and is replaced by the conductance step and plateau. At the highest temperature of $2.5 \mathrm{~K}$ measured in the dilution refrigerator, the peaks in the open regime have practically disappeared, while those in the tunneling regime show a trace existence. The influence of the source-drain voltage $V_{d s}$ on the regular $G$ pattern has also been studied and is shown in Fig. 7. Notice that the effect of increasing $V_{d s}$ is similar to that of temperature. The oscillations in $G$ are found to practically fade out and be replaced by the conductance step and plateau when $V_{d s}$ is increased to a rms value of $700 \mu \mathrm{V}$. 


\section{THEORETICAL TREATMENT}

\section{A. Cavity potential}

A realistic, theoretical description of ballistic electron transport through a cavity, such as the stub of an EST, must take into account the electrostatic potential inside the cavity since it determines the actual shape of the conducting channel. Accordingly, we have calculated the electrostatic potential created in the plane of a 2DEG situated at a distance $d=80 \mathrm{~nm}$ below the surface, at $z=0$, of a two-gate EST, defined by two surface Schottky gates with voltages $V_{T}$ and $V_{B}$, when $S 1, T$, and $S 2$ are connected together, cf. Fig. 1. The distance between the gates at entrance and exit is $2 w=250 \mathrm{~nm}$, the bottom gate $G_{B}$ is flat, while the top gate $G_{T}$ contains the stub-like opening of width $2 w$ and of length $L=300 \mathrm{~nm}$. This value of $d$ and the lithographic dimensions correspond to the experimental device described above, although the present model of just two gates is somewhat idealized but necessary for simplifying the calculations. The potential $\varphi(x, y, z)$ has been calculated from the Laplace equation in the semi-space $z>0$, with Dirihlet boundary conditions $\varphi(x, y, 0)=V_{i}$ on the $i$-th gate region $(i=T, B)$ and Newmann boundary conditions $\partial \varphi(x, y, z) /\left.\partial z\right|_{z=0}=4 \pi e n_{2 D} / \epsilon$ at the exposed surface region; $n_{2 D}$ is the electron concentration in the $2 \mathrm{D}$ gas in the absence of depletion and $\epsilon$ is the dielectric constant. The last boundary condition expresses the so-called "frozen surface model" in which the electric charge at the exposed surface is constant; this model appears appropriate at low temperatures and is often used in theoretical calculations [8]. To make our model finite in the $x$ direction, we choose a length $l=L$ and use the boundary condition $\partial \varphi(x, y, z) /\left.\partial x\right|_{x= \pm l}=0$. We have also assumed that the concentration of the ionized donors in the doping region between the surface and the $2 \mathrm{D}$ gas plane is equal to a sum of the $n_{2 D}$ and surface charge concentration and is not changed appreciably when the voltages are applied to the gates.

In Fig. 8 we present the resulting contour plot of the potential $\varphi(x, y, d)$; since $\varphi(-x, y, z)=\varphi(x, y, z)$, we show $\varphi$ only for the right half of the stub. Although we do not take into account the free-electron charge in the plane $z=d$, in order to avoid the heavily involved self-consistent calculations, we expect that the screening effect due to these electrons will not change the shape of the equipotential lines considerably but would cause at most a flattening of the bottom of the potential distribition. As a result, we expect the shape of the conducting channel to follow, more or less, the calculated equipotential lines. This enables us to draw the following important qualitative conclusions.

i) The shape of the cavity inside the stub region does not follow that defined by the gate edges and is not rectangular as has been assumed in previous pertinent theoretical works.

ii) The width of the cavity is close to the lithographic width of the stub, and since the Fermi wavelength at $E_{F} \simeq 8 \mathrm{meV}$ is about $53 \mathrm{~nm}$, which is considerably less than the lithographic stub width $2 w$, the cavity accommodates not just one longitudinal mode, as has been frequently assumed, but several modes.

iii) When the width of the narrowest part of the conducting channel, in our model at $x=l$, is small compared to the lithographic one $2 w$ of the wire, the length of the cavity at $x=0$ is considerably smaller than the lithographic length $L$.

iv) The length of the cavity is even smaller when the upper gate voltage $V_{T}$ is more negative $\left(V_{T}<V_{B}\right)$ so that there is an overall shift of the conducting channel towards the bottom 
gate. In going beyond the two-gate model towards the four-gate device shown in Fig. 1, it is reasonable to expect that when the top gate voltage is more negative than that of the side gates, the height of the cavity decreases, while in the opposite case it should increase.

In the following we use this qualitative information to appropriately model the shape of the conducting channel in the four-gate EST and calculate the electron transmission through the cavity.

\section{B. Model of the cavity and numerical method}

We model the conducting channel of the device in Fig. 1 with a 2D waveguide having hard-wall boundaries described, in an obvious notation, by the functions

$$
\begin{array}{r}
y_{\text {bot }}(x)=-y_{\text {wire }}(x), \quad y_{\text {wire }}(x)=W /[1+\exp ((-x+r) / \beta)]+W /[1+\exp ((x+r) / \beta)], \\
y_{\text {top }}(x)=y_{\text {wire }}(x)+a+y_{\text {cav }}(x), \quad y_{\text {cav }}(x)=h \exp \left(-x^{2} / b^{2}\right) .
\end{array}
$$

We describe the cavity with the Gaussian function $y_{\text {cav }}(x)$ since it gives us the most relevant elementary-function approximation of the equipotential lines shown in Fig. 8. The function $y_{\text {wire }}(x)$ describes the transition from the constricted region near $x=0$ to the $2 \mathrm{D}$ reservoirs at $x= \pm \infty$. Here we set $\beta=W / 4$ to model the square-angle opening of the conducting channel of the experimental device (Fig. 1). The parameter $W$, which describes the semiwidth of the channels away from the constriction and must be large enough, is chosen as $W=w$. For this value of $W$, the channels away from the constriction already accommodate about 10 transverse modes and can be effectively treated as 2D leads. The remaining parameter $r$ is chosen, by inspection, as $r=w+l+3 \beta$, where $l$ is the lithographic length of the QPCs; this gives a more or less suitable correspondence between the outer parts of the conducting channel and the gate corners. The resulting shape of the conducting channel, together with the lithographic gate layout, is shown in Fig. 9.

To determine the transmission coefficients of electron waves through the device, we solved numerically the 2D Schrödinger equation

$$
-\frac{\hbar^{2}}{2 m}\left(\frac{\partial^{2}}{\partial x^{2}}+\frac{\partial^{2}}{\partial y^{2}}\right) \Psi(x, y)+[U(x, y)-\varepsilon] \Psi(x, y)=0
$$

using the following expansion for the wave function [9]

$$
\Psi(x, y)=\sum_{n} \psi_{n}(x) \chi_{n}(x, y), \quad \chi_{n}(x, y)=\sqrt{\frac{2}{Y(x)}} \sin \left[\frac{\pi n}{Y(x)}\left(y-y_{b o t}(x)\right)\right],
$$

where $Y(x)=y_{\text {top }}(x)-y_{\text {bot }}(x)$ is the $x$-dependent channel width. The basis functions $\chi_{n}(x, y)$ already satisfy the boundary conditions for hard-wall confinement. Substituting Eq. (4) into Eq. (3) leads to the 1D matrix equation for $\psi_{n}(x)$

$$
\left[\frac{d^{2}}{d x^{2}}-\left(\frac{\pi n}{Y(x)}\right)^{2}+k^{2}\right] \psi_{n}(x)+\sum_{m}\left[2 B_{n m}(x) \frac{d}{d x}+C_{n m}(x)-K_{n m}(x)\right] \psi_{m}(x)=0
$$

here $k^{2}=2 m \varepsilon / \hbar^{2}$ and 


$$
\begin{array}{r}
B_{n m}(x)=\int_{y_{b o t}(x)}^{y_{t o p}(x)} d y \chi_{n}(x, y) \frac{\partial}{\partial x} \chi_{m}(x, y), \\
C_{n m}(x)=\int_{y_{b o t}(x)}^{y_{t o p}(x)} d y \chi_{n}(x, y) \frac{\partial^{2}}{\partial x^{2}} \chi_{m}(x, y), \\
K_{n m}(x)=\frac{2 m}{\hbar^{2}} \int_{y_{b o t}(x)}^{y_{t o p}(x)} d y \chi_{n}(x, y) U(x, y) \chi_{m}(x, y) .
\end{array}
$$

Since we assume $U(x, y)=0$ far away from the constriction, all parameters defined by Eqs. (6) depend on $x$ only in the constriction region. We choose $x_{\max }$ and $x_{\min }$ far enough from the constriction and discretize Eq. (5) on a $N+1$-point grid according to $x=x_{i}=$ $x_{\min }+i s, s=\left(x_{\max }-x_{\min }\right) / N$. The resulting finite-difference equation for $\psi_{m}\left(x_{i}\right)$ is solved subject to the boundary conditions $A_{n m}(1) \psi_{m}\left(x_{1}\right)+A_{n m}(0) \psi_{m}\left(x_{0}\right)=A_{n}^{\alpha}$ and $A_{n m}(N-$ 1) $\psi_{m}\left(x_{N-1}\right)+A_{n m}(N) \psi_{m}\left(x_{N}\right)=0$, appropriate to a wave, in state $\alpha$, incident from the left side. Since $\chi_{n}(x, y)$ are the exact normalized eigenfunctions of the problem at $x= \pm \infty$, the boundary matrices $A_{n m}$ are diagonal $A_{n m}(1)=A_{n m}(N-1)=\delta_{n m}, A_{n m}(0)=A_{n m}(N)=$ $-\delta_{n m} \exp \left(-i p_{n} s\right)$, while $A_{n}^{\alpha}=\delta_{n \alpha}\left[\exp \left(i p_{n} s\right)-\exp \left(-i p_{n} s\right)\right]$. In these matrix expressions we introduced the longitudinal quantum number

$$
p_{n}=\sqrt{k^{2}-(\pi n / Y(\infty))^{2}},
$$

which can be either real or imaginary $\left(\operatorname{Im} p_{n}>0\right)$; in the latter case the waves are evanescent in the leads. Far away from the constriction $p_{n}$ is the longitudinal momentum in the leads.

The ballistic conductance $G$ at zero temperature is given by the multichannel LandauerBüttiker formula

$$
G=\frac{2 e^{2}}{h} \sum_{\alpha \alpha^{\prime}}\left|T_{\alpha \alpha^{\prime}}\right|^{2} \frac{p_{\alpha^{\prime}}}{p_{\alpha}} .
$$

The transmission amplitude $T_{\alpha \alpha^{\prime}}$ in Eq. (8) is equal to $\psi_{\alpha^{\prime}}\left(x_{N}\right)$ for the problem with the incident wave in state $\alpha$ and $\varepsilon=E_{F}$. The sum runs over all propagating states (for which $p_{\alpha}$ are real). The generalization of Eq. (8) to finite temperatures is straightforward, see, e.g., Eq. (7) in Ref. 10. The actual number $M$ of transverse subbands involved in the numerical calculations is determined by the condition that further increase of $M$ does not produce any perceptible change of the calculated wave functions and transmission coefficients. For the calculations described below it is sufficient to take $M$ between 10 and 15; this results in a reasonably short calculation time.

\section{Numerical results}

In order to decrease the number of the unknown parameters, we restricted ourselves to the flat-band approximation, $U(x, y)=0$, in all calculations. As a result, we have only the three geometrical parameters $a, h$ and $b$, which are assumed to be controlled by the gates. The first of them, $a$, characterizes the width of the constriction in its narrowest parts, although it is somewhat smaller than the constriction width, cf. Eqs. (1) and (2). Assuming that the depletion of the 2DEG by the gates follows a linear law, which is confirmed by 
our experimental studies of the conductance quantization in a single constriction, we can directly associate a change of the value of $a$ with a change of the gate voltages. As for $h$ and $b$, they describe mostly the shape of the cavity formed in the stub region and are related, respectively, to its height and width. It is convenient to measure all these parameters in units of the cut-off length $a_{0}=\hbar \pi / \sqrt{2 m E_{F}}$, which is the width of the hard-wall quantum wire, when it stops conducting, and is equal to $26.5 \mathrm{~nm}$ for $E_{F}=8 \mathrm{meV}$, a value of $E_{F}$ used in all calculations, and GaAs effective mass $m=0.067 m_{e}$.

Varying the bottom gate voltage $V_{B}$, in our model, means simply changing the parameter $a$ while keeping $h$ and $b$ constant. This describes the case when the lower (bottom) boundary of the conducting channel is shifted linearly by $V_{B}$, while the upper boundary remains insensitive to this voltage because of screening by the electron gas inside the channel. These assumptions are supported by previous calculations of the potential distribution in homogeneous, along $x$ axis, split-gate structures [8]. The calculated dependence of the conductance on $a / a_{0}$, in the range of the first and second plateau, for two values of $h$ and $b=w$ is shown in Fig. 10. The following qualitative features are evident: for small $h$ the transmission pattern shows narrow, almost equally spaced minima of resonance reflections of similar shape: we call this the "regular" pattern. The number of the minima on the first plateau (5-7) is consistent with the experimentally observed number (Fig. 5, solid curve). As $h$ increases ( $V_{T}$ less negative), the oscillations become irregular and show broad troughs, on which are superimposed the closely spaced resonances, and the average conductance is considerably smaller than that of the shorter cavity. These results are consistent with experimental observations (Fig. 5, dotted curve). Results for a narrower cavity show a similar but less regular pattern.

The situation is more complex when the top gate voltage $V_{T}$ is varied. Making $V_{T}$ less negative obviously leads to an increase of $h$, and, as mentioned earlier, it also widens the constriction. To describe this situation $a$ and $h$ should be varied. Very likely the cavity width $b$ also changes in this situation, but we expect this change to be small and make the following assumptions in order to generate the numerical results: $b$ remains unchanged while $h$ varies linearly as $h=h_{0}+v a$ as $V_{T}$ is changed. Since the distance of the top gate from the highest part of the cavity is smaller, but not much smaller, than its distance from the narrowest part of the constriction, we expect $v$ to be positive but not much larger than unity.

The dependence of $G$ on $a / a_{0}$, for fixed $b=w$ and different $h_{0}$ and $v$, is shown in Fig. 11 for the region of the first plateau. The curves marked (1) and (2) are obtained, respectively, with the $h_{0}=1.2 a_{0}, v=1$, for (1), and $h_{0}=0.6 a_{0}, v=2$ for (2). The parameters are chosen in such a way that at the beginning of the plateau $\left(a / a_{0} \simeq 0.6\right)$ the cavity height is equal to $1.8 a_{0}$ for both curves. Curve (1), with smaller $v$, shows an oscillatory $G$ with regular minima, similar to those of curve (2) in Fig. 10 for constant $h$. But there is also a difference: the average conductance shows a small depression near the end of the plateau. As $v$ increases (curve (2)), the depression becomes more pronounced and broad, spreading over the second half of the plateau. Similar results, but with a less regular oscillatory pattern, are obtained for cavities with larger $h$, e.g., with $h \simeq 2.6 a_{0}$ at the beginning of the plateau. Also similar qualitative features have been obtained for narrower cavities $(b=0.75 w)$.

We do not discuss separately the case when the side-gate voltage $V_{S}$ is varied, with $h$ and $a$ changing simultaneously, since we expect the effect to be the same as when $V_{T}$ changes. 
The experimental results of Figs. 3 and 4 show no qualitative difference between the two cases and confirm this assessment.

\section{DISCUSSION AND CONCLUSIONS}

Comparison of the experimental and theoretical results shows that the qualitative behavior of the ballistic conductance, as a function of different gate voltages, is the same in either case. This indicates that our choice of parameters and the assumptions about their variation with gate voltages, supported in part by the solution of the electrostatic problem, represent fairly the experimental situation. Below we discuss this in more detail.

When we make $V_{S}$ and $V_{T}$ more negative than $V_{B}$, the conducting channel is shifted towards the bottom gate and the cavity height $h$ decreases. In this situation we obtain rather regular oscillations of the conductance as a function of bottom, top or side gate voltages. The minima correspond to resonant reflections of the electron waves from quasibound states in the cavity [11]. Variation of one of the gate voltages sweeps the levels of the quasibound states through the Fermi level $E_{F}$ and results in a resonance minimum each time $E_{F}$ coincides with one of them. The regularity in shape and spacing of these minima, for cavities with small heights, follows from the fact that these minima originate from the same set of quasibound states. For confirmation, we calculated again curves (1) in Figs. 10 and 11 with just two transverse cavity modes in the expansion (4); in the region of the first plateau the first mode is transmitted through the constrictions, while the second one is quasibound in the cavity. Comparing these results with the curves (1) of Figs. 10 and 11, we find that all regular resonances occuring on the first plateau appear also in this simplified calculation and their shapes are very similar. We, therefore, conclude that the regular resonance reflection pattern results from quasibound states associated with the second transverse mode and each of them characterized by a proper wavenumber that expresses longitudinal quantization along the $x$ direction. Higher transverse quantization states bring additional resonant features and make the dependence of the conductance on the gate voltages less regular. The experimental results of Figs. 3-5 (solid curves) show well-defined regularly spaced minima in $G$ and fully corroborate this analysis. Note that the number of the minima obtained from the theory is about the same as that observed experimentally; this means that the cavity width is really close to the lithographic width $2 w$ of the stub, as we assumed in our model. The experimental dependences of the conductance on the top, side, and bottom gate voltages are similar and show almost the same number of oscillations. This is in agreement with our assumption that we are dealing with a wide cavity of small height. For the same $h$ the minima are narrower for wider cavities since in such a case the upper boundary of the cavity is smoother and the electron motion through the cavity is more adiabatic. In terms of our model this means that the derivative $d y_{c a v}(x) / d x$ is smaller. Experimentally, a broadening

of the cavity without changing its height can be achieved by applying less negative voltages to the side gates.

When we make $V_{S}$ and/or $V_{T}$ less negative compared to the values of the "regular" case discussed above, the channel widens and the cavity height $h$ increases. In this "irregular" case the situation is modified as follows. i) More quasibound states occur and influence the resonant reflection, ii) The coupling between transmitted and quasibound states increases due to the increase of the non-adiabaticity. Both these factors should lead to a decrease 
of the average conductance $G$. This decrease of $G$ is clearly seen in the theoretical results, cf. Figs. 10-11. Besides, the theory shows that the behavior of $G$ as a function of different gate voltages has a qualitative difference: the variation of $V_{B}$ produces broad minima and depression of the average $G$ over the entire plateaus while the variation of $V_{T}$ or $V_{S}$ gives narrower minima and the average $G$ has pronounced troughs near the ends of the plateaus. Our theoretical calculations show that the troughs are more pronounced when the height $h$ is larger and when it increases faster with the opening of the constriction. A simple explanation is as follows. Sweeping $V_{T}$ or $V_{S}$ towards less negative values along the conductance plateau substantially increases $h$ and leads to a departure from the short-cavity, "regular" pattern to the "irregular" one of a long-cavity. Therefore, the averaged $G$ decreases at the end of the first plateau and then increases, when the second transverse mode is allowed in the constriction, reaching the next plateau. If the initial value of $h$ is already large, we need a lesser increase in $h$ in order to move to the long-cavity regime; then the decrease of the average $G$ appears earlier and gives rise to a broader trough. The experimentally observed behavior of $G$, cf. dashed curves in Figs. 3-5, shows pronounced troughs and a decrease in its average value in agreement with the above interpretation. We emphasize that the appearance of these troughs in $G$ is a very common and reproducible feature of the stubbed quantum devices studied in this work.

Possible EST applications require a regular, periodic dependence of the conductance on the gate voltages. The initial idea ${ }^{1}$, followed in subsequent theoretical works, was to use a narrow cavity, containing one quantized state, or mode, in the $(x)$ transport direction, and control the conductance through it by changing the height of the cavity by a top-gate voltage. However, from our results it is clear that real EST devices do not satisfy these conditions because the electrostatic potential created by the gates is rounded near the corners, cf. Fig. 8. Moreover, for a real device, as this work shows, if the cavity is long enough, the dependence of the conductance on the gate voltages is rather irregular. We have shown that it is possible to obtain a short-length cavity by a proper choice of the gate voltages that is wide in the transport direction. The conductance through such a cavity shows a regular pattern of resonant reflection minima associated with the quasibound states in it that result from longitudinal quantization.

\section{ACKNOWLEDGMENTS}

One of us (P. D.) gratefully acknowledges the award of a Senior Research Associateship by the National Research Council, Washington, DC. The work of P. V. was supported by the Canadian NSERC Grant No. OGP0121756. 


\section{REFERENCES}

$\dagger \quad$ E-mail: debray@spec.saclay.cea.fr

* E-mail: raichev@zinovi@lab2.kiev.ua

• E-mail: takis@boltzmann.concordia.ca

[1] F. Sols, M. Macucci, U. Ravaioli, and K. Hess, Appl. Phys. Lett. 54, 350 (1989); J. Appl. Phys. 66, 3892 (1989); A. B. Fowler, US Patent No 4,550,330 (29 October 1985); S. Datta, Superlatt. Microstruct. 6, 83 (1989).

[2] C. M. Marcus, A. J. Rimberg, R. M. Westervelt, P. F. Hopkins, and A. C. Gossard, Phys. Rev. Lett. 69, 506 (1992); M. Persson, J. Pettersson, B. von Sydow, P. E. Lindelof, A. Kristensen, and K. -F. Berggren, Phys. Rev. B 52, 8921 (1995); I. V. Zolulenko, R. Schuster, K. -F. Berggren, and K. Ensslin, ibid 55, R10229 (1997); K. Aihara, M. Yamamoto, and T. Mizutani, Jpn. J. Appl. Phys., Letters 31, L916 (1992).

[3] K.-F.Berggren and Z.-L.Ji, Phys. Rev. B 43, 4760 (1991); H. Wu, D. W. L. Sprung, J.Martorell, and S. Klarsfeld, ibid. 44, 6351 (1991); Y. Takagaki and D. K. Ferry, ibid. 45, 6715 (1992); W. Porod, Z. Shao, and C. S. Lent, ibid. 48, 8495 (1993); P. S. Deo and A. M. Jayannavar, ibid. 50, 11629 (1994); J. Appenzeller, Ch. Schroer, Th. Schäpers, A. v. d. Hart, A. Förster, B. Lengeler, and H. Lüth, ibid. 53, 9959 (1996); J. Wang and H. Guo, ibid. 54, R11090 (1996); K. Nikolic and R. Sordan, ibid. 58, 9631 (1998); P. Debray, R. Akis, P.Vasilopoulos, and J. Blanchet, Appl. Phys. Lett. 66, 3137 (1995).

[4] R. Akis, P.Vasilopoulos, and P. Debray, Phys. Rev. B 56, 9594 (1997).

[5] P. Debray, O. E. Raichev, M. Rahman, R. Akis, and W. C. Mitchell, Appl. Phys. Lett. 74, 768 (1999).

[6] J. H. Davies, I. A. Larkin, E. V. Sukhorukov, J. Appl. Phys. 77, 4504 (1995).

[7] K.-F.Berggren and Z.-L.Ji, Phys. Rev. B 43, 4760 (1991).

[8] L. I. Glazman and I. A. Larkin, Semicond. Sci. Technol. 6, 32 (1991); D. B. Shklovskii, B. I. Shklovskii and L. I. Glazman, Phys. Rev. B 46, 4026 (1992).

[9] S. Kasai, K. Mitsutake and A. Okiji, Journ. Phys. Soc. Japan 60, 1679 (1991).

[10] R. Akis, P. Vasilopoulos, and P. Debray, Phys. Rev. B 52, 2805 (1995).

[11] J. Wang, Y. Wang, and H. Guo, Appl. Phys. Lett. 65, 1793 (1994); J. Price, Phys. Rev. B 48, 17301 (1993). 


\section{FIGURES}

FIG. 1. Schematic drawing of an EST device. Lithographic dimensions: $L=0.30 \mu \mathrm{m}$, $2 W=0.25 \mu \mathrm{m}$. S1, T, S2, and B are surface Schottky gates. The area enclosed between the dashed lines defines the conducting channel.

FIG. 2. Scanning electron micrograph of a fabricated EST device. Lighter areas indicate the Schottky gates.

FIG. 3. Conductance as a function of the top gate voltage $V_{T}$ with $V_{S 1}=-860 \mathrm{mV}$ and $V_{S 2}=-964 \mathrm{mV}$. The solid and dotted curves correspond to $V_{B}=-765 \mathrm{mV}$ and $V_{B}=-780 \mathrm{mV}$, respectively. The arrows indicate locations of broad depressions in $G$.

FIG. 4. Conductance as a function of the side gate voltage $V_{S}\left(V_{S}=V_{S 1}=V_{S 2}+104 \mathrm{mV}\right)$ at fixed $V_{B}$ and $V_{T}$. The solid curve was obtained at $V_{T}=-1400 \mathrm{mV}$ and $V_{B}=-755 \mathrm{mV}$. The dotted curve corresponds to $V_{T}=-800 \mathrm{mV}$ and $V_{B}=-790 \mathrm{mV}$. The arrow shows the position of a broad depression in $G$.

FIG. 5. Conductance as a function of the bottom gate voltage $V_{B}$ with $V_{S 1}=-860 \mathrm{mV}$ and $V_{S 2}=-964 \mathrm{mV}$ at fixed $V_{T}$. The solid and dotted curves correspond to $V_{T}=-1085 \mathrm{mV}$ and $V_{T}=-635 \mathrm{mV}$, respectively. The arrows indicate locations of broad depressions in $G$.

FIG. 6. Conductance as a function of the top gate voltage $V_{T}$ with $V_{S 1}=-860 \mathrm{mV}, V_{S 2}=-964$ $\mathrm{mV}$, and $V_{B}=-765 \mathrm{mV}$ at different temperatures.

FIG. 7. Conductance as a function of the top gate voltage $V_{T}$ with $V_{S 1}=-860 \mathrm{mV}, V_{S 2}=-964$ $\mathrm{mV} . V_{B}=-765 \mathrm{mV}$ for different source-drain voltages.

FIG. 8. Contour plot of $\phi(x, y, z)$ created in the plane of the $2 \mathrm{DEG}$, at $z=d=80 \mathrm{~nm}$, of a two-gate EST for (a) $V_{B}=V_{T}$ and (b) $V_{B}-V_{T}=0.2 \mathrm{~V}$. The interval between equipotential lines is $6.6 \mathrm{mV}$ in (a) and $8.4 \mathrm{mV}$. The thick solid lines show the edges of the gates.

FIG. 9. Model of the conducting channel for a four-gate EST (Figs. 1 and 2). The area enclosed between the traces $y_{t}$ and $y_{b}$ defines the shape and size of the conducting channel under

gate biases that was used in our calculations. The solid straight lines are the lithographic edges of the Schottky gates S1, T, S2, and B.

FIG. 10. Conductance as function of $a / a_{0}$ for two different heights of the stub cavity when its width is equal to the lithographic width $(b=w)$ : (1) $h=2 a_{0} ;(2)$ (shifted for clarity) $h=3 a_{0}$. This corresponds to the experimental condition when $V_{B}$ is varied with $V_{T}$ and $V_{S}$ constant.

FIG. 11. Conductance as function of $a / a_{0}$ for $b=w$ and $h=h_{0}+v a$. (1) $h_{0}=1.2 a_{0}, v=1$; (2) $h_{0}=0.6 a_{0}, v=2$. This corresponds to the experimental condition when $V_{T}$ is varied with $V_{B}$ and $V_{S}$ constant. 


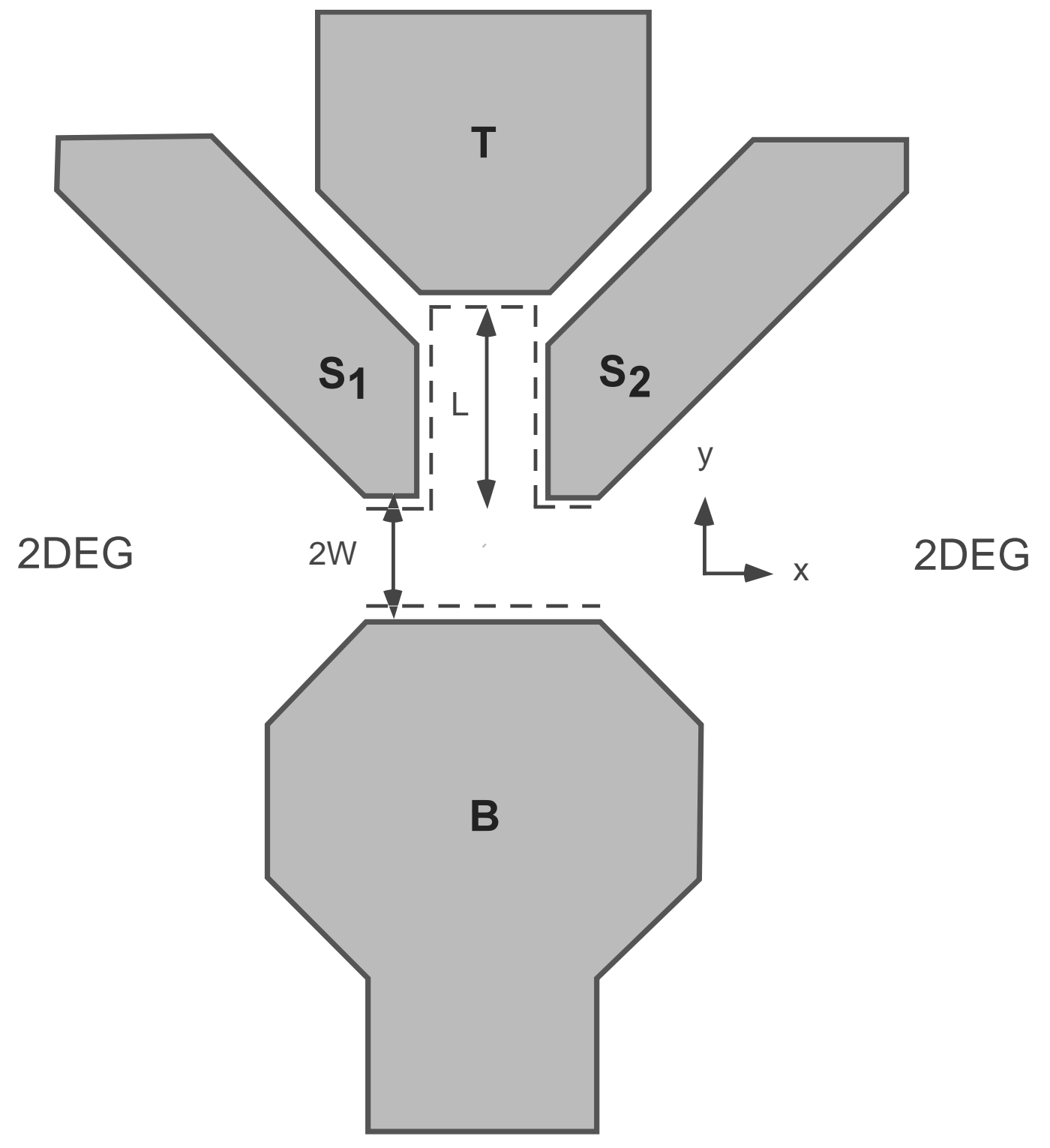

Fig. 1.

P. Debray et al. 


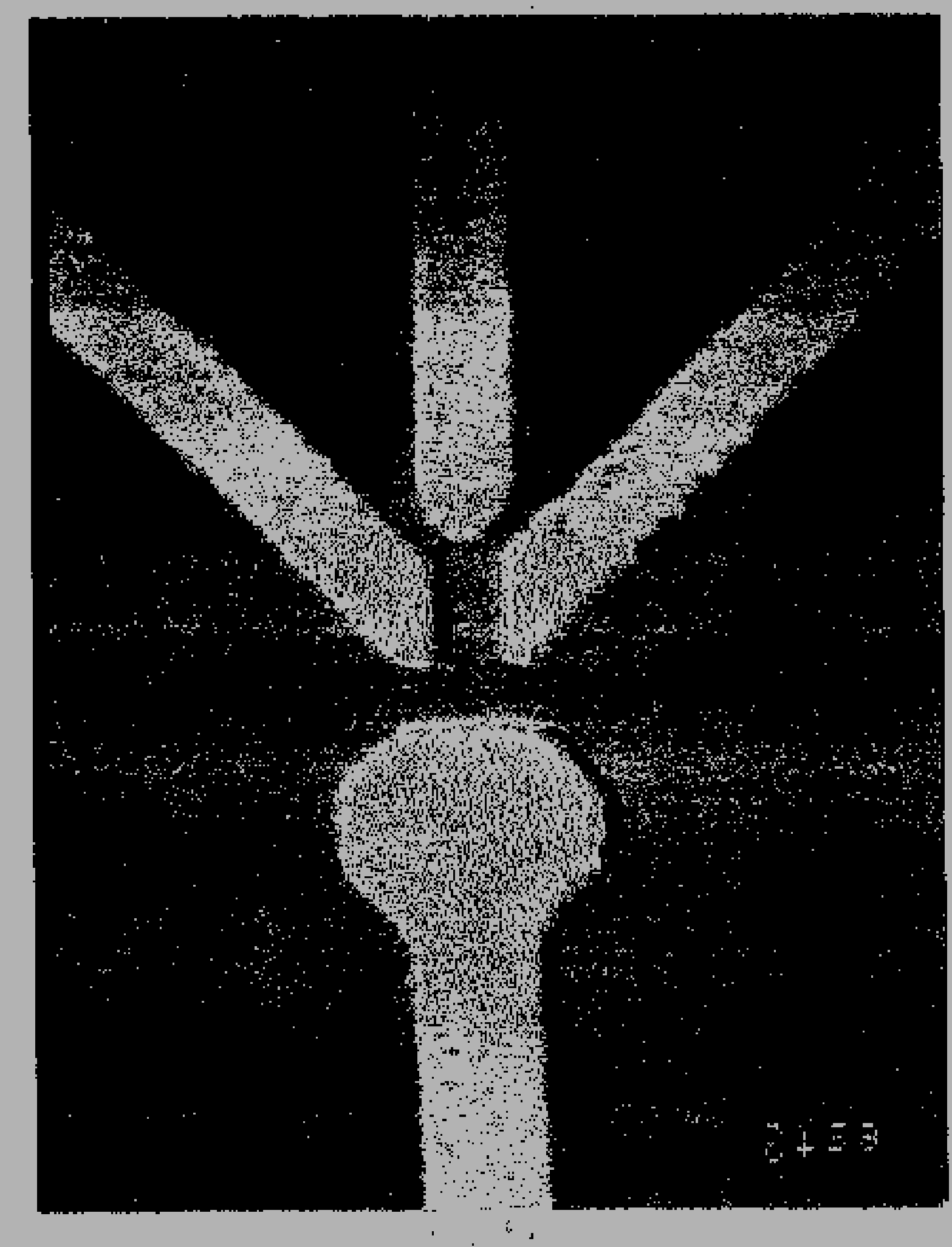

Fig. 2

P. Debray et al. 


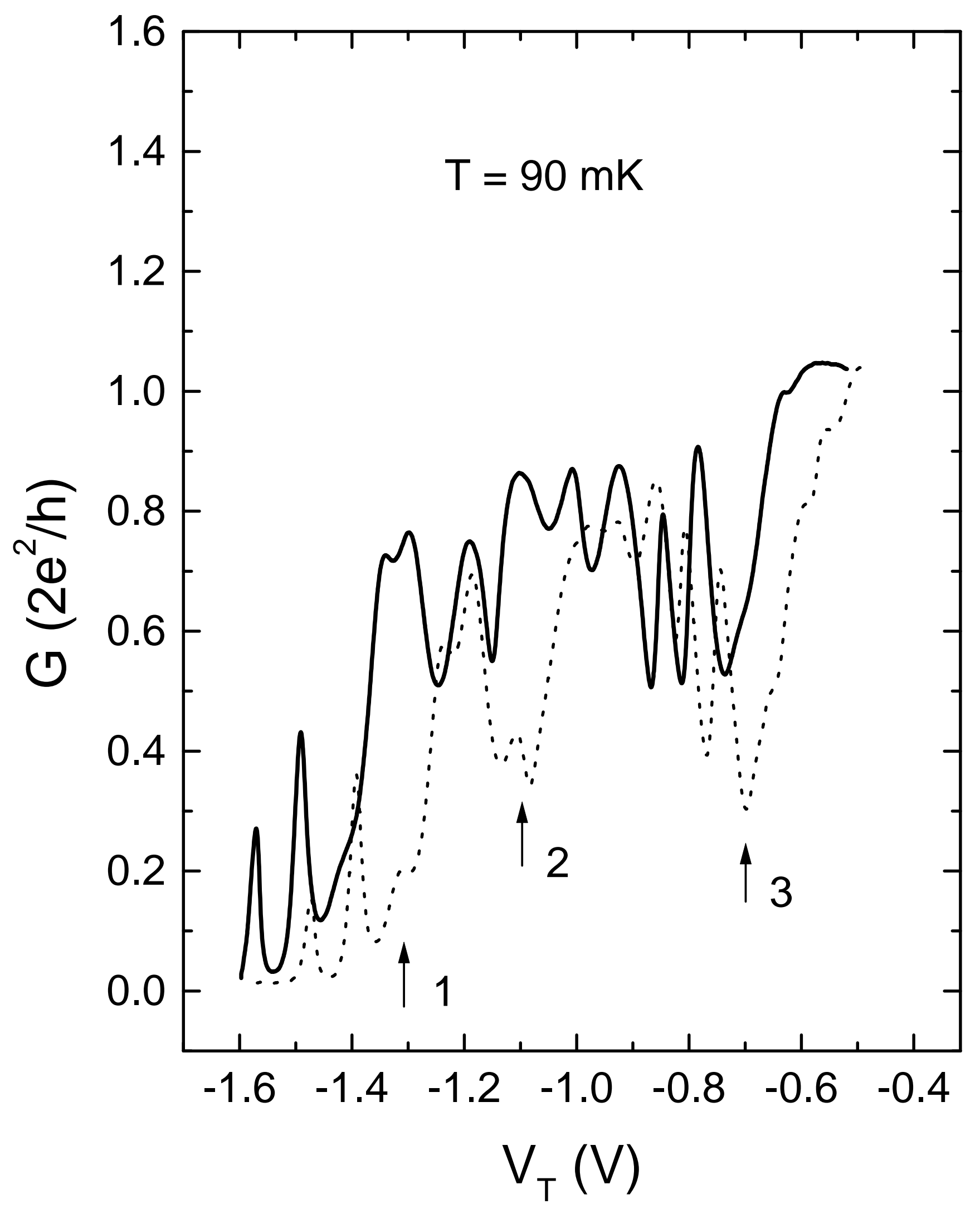

Fig. 3 P. Debray et al. 


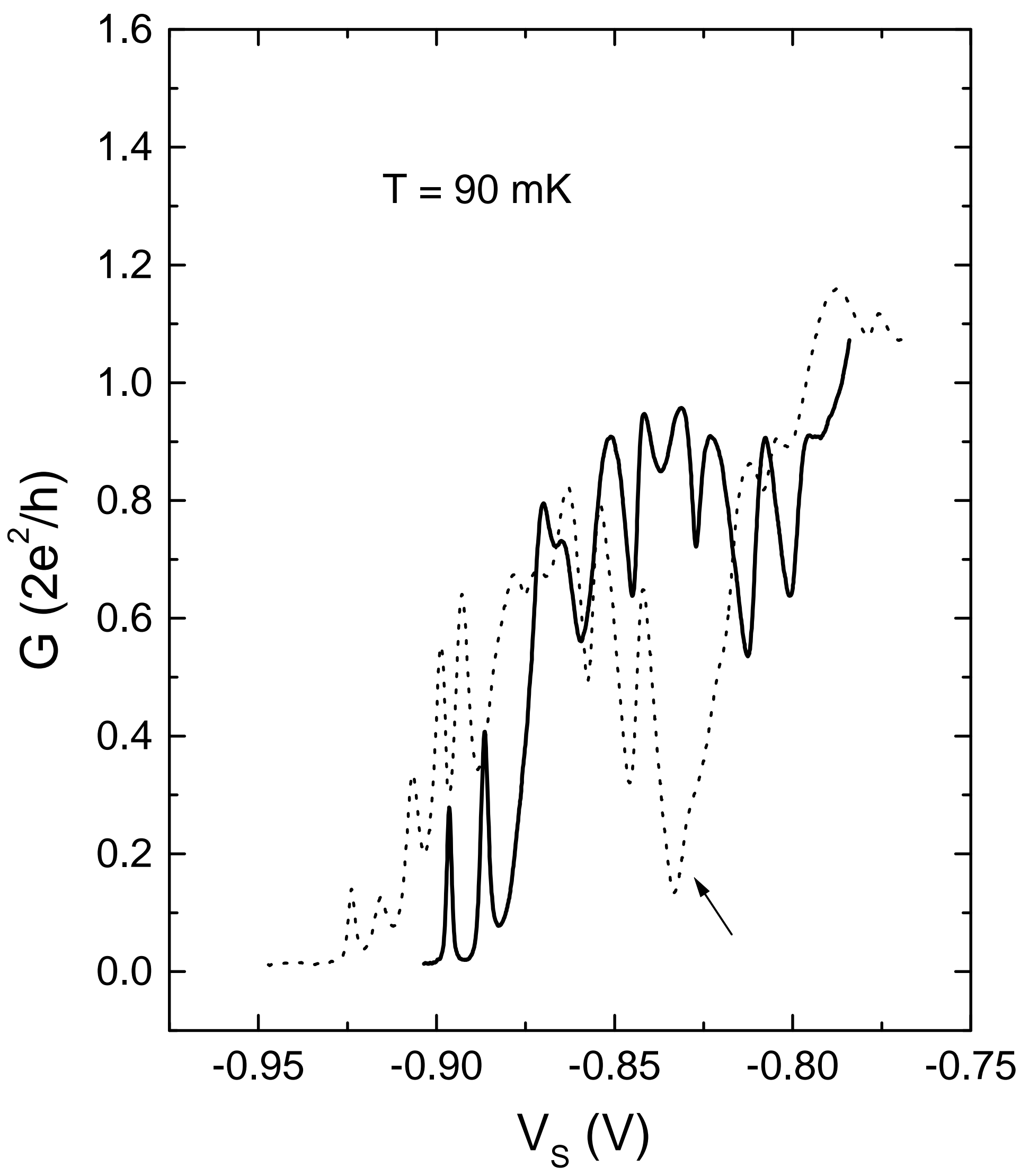

Fig. 4. P. Debray et al. 


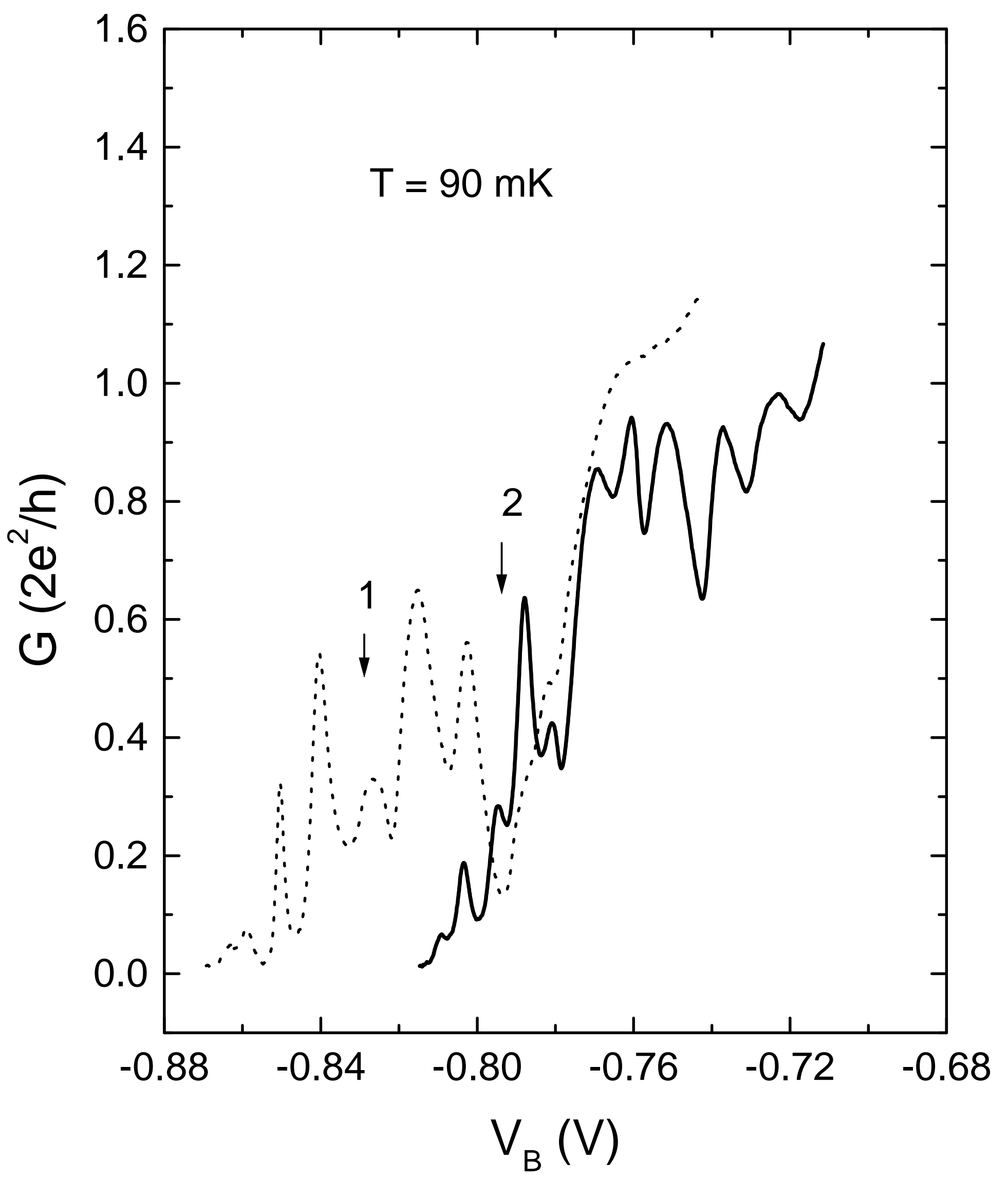

Fig. 5. P. Debray et al. 


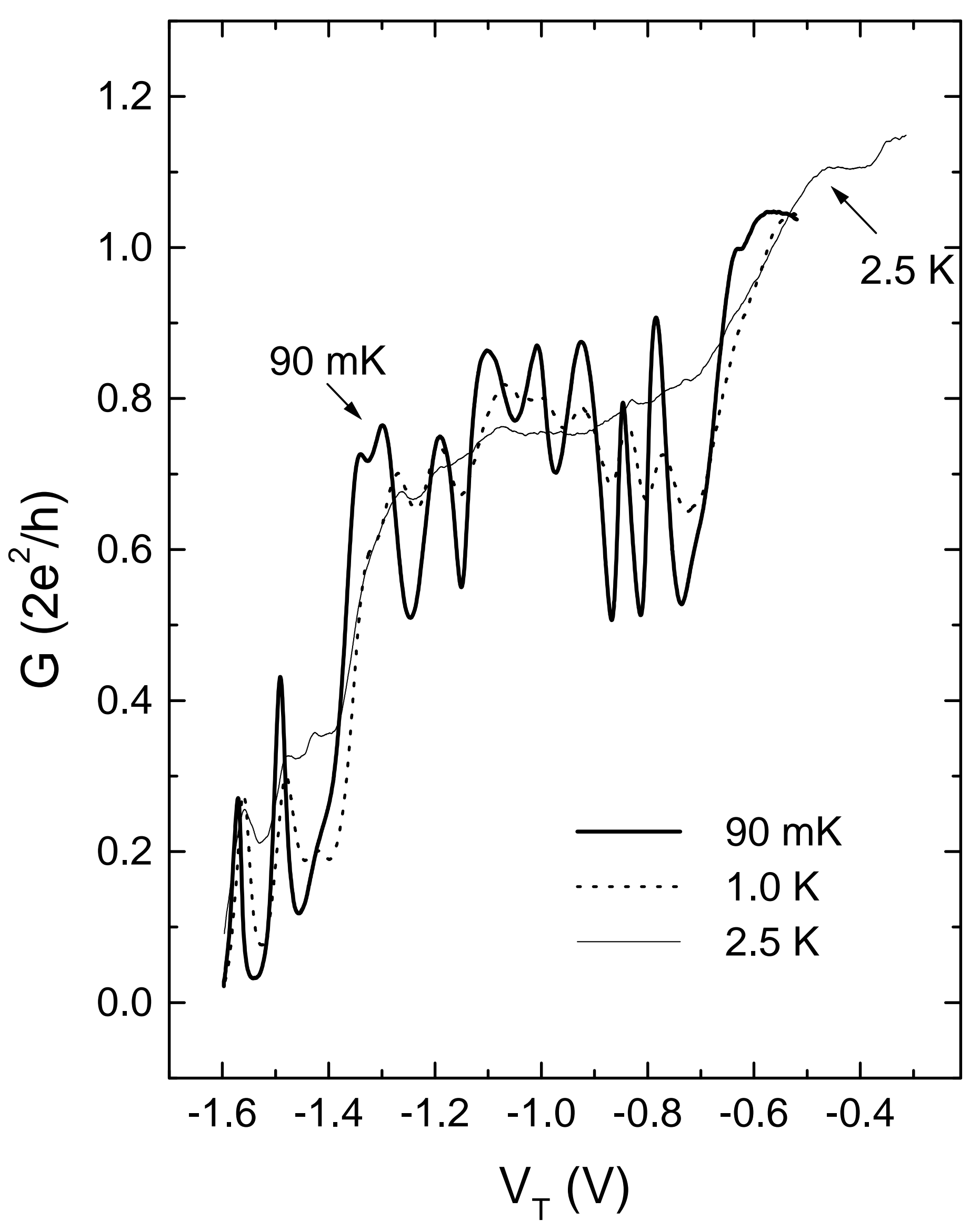

Fig. 6. P. Debray et al. 


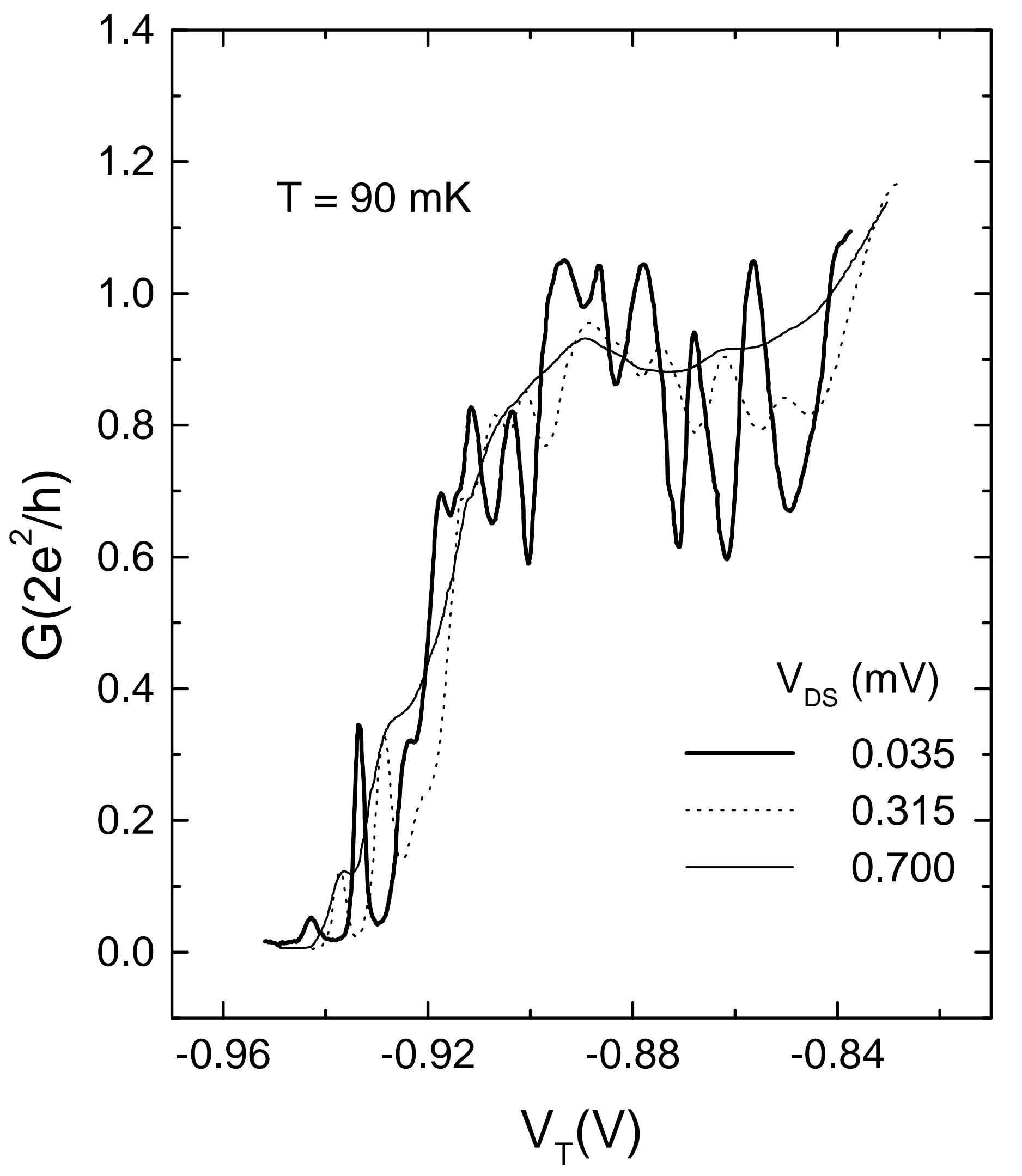

Fig. 7. P. Debray et al. 

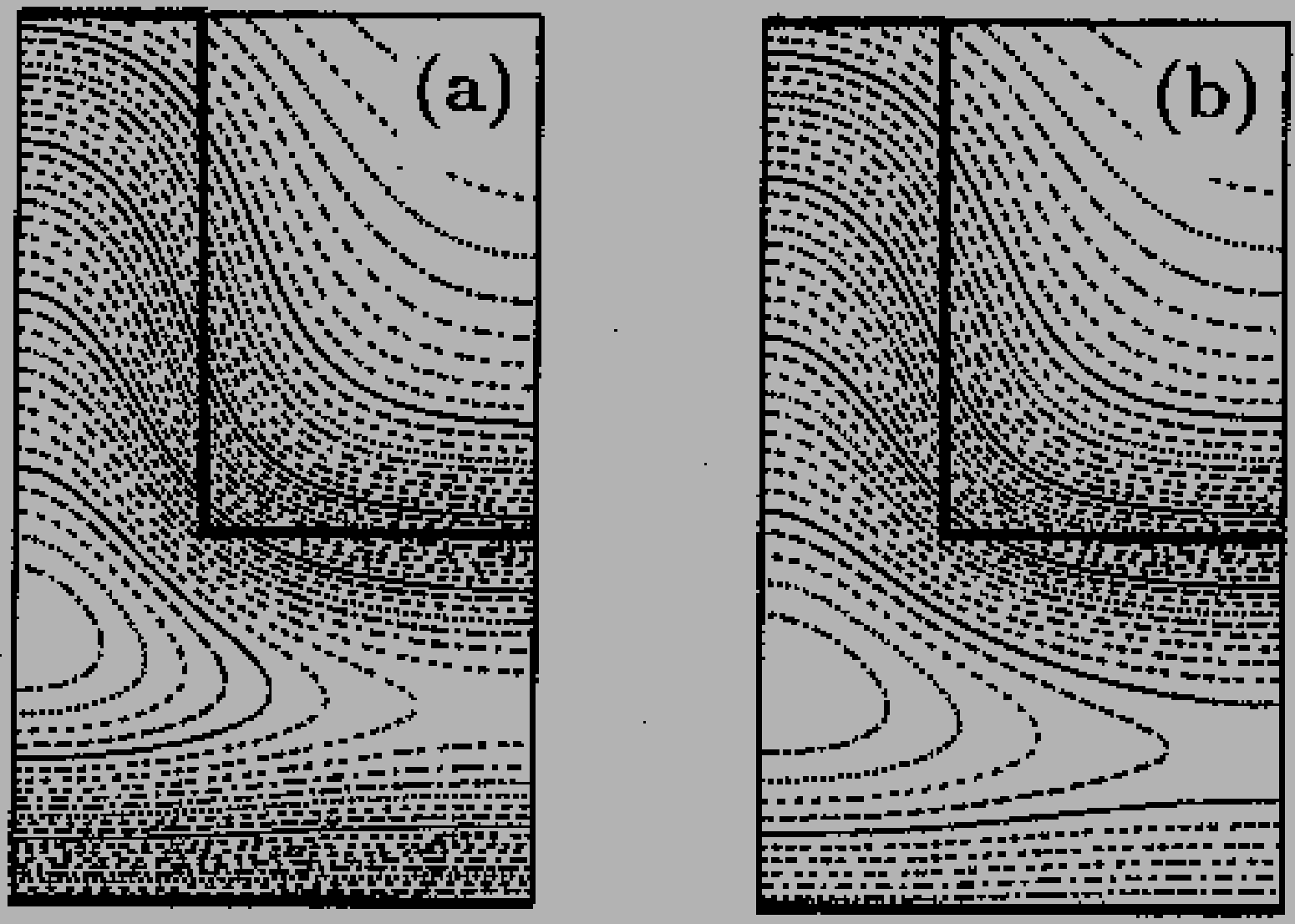

Fig. 8

P. Debray et al. 


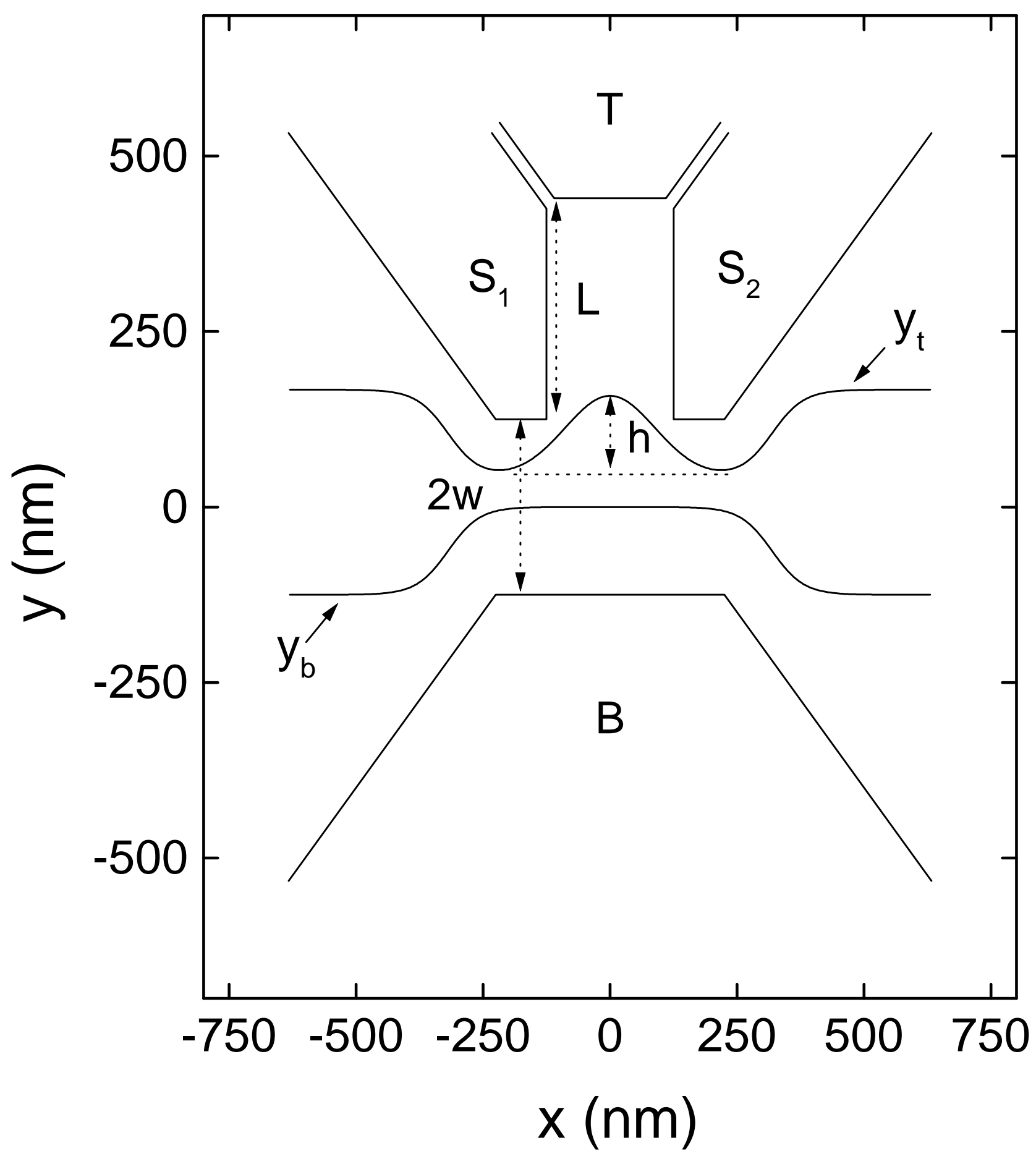

Fig. 9. P. Debray et al. 


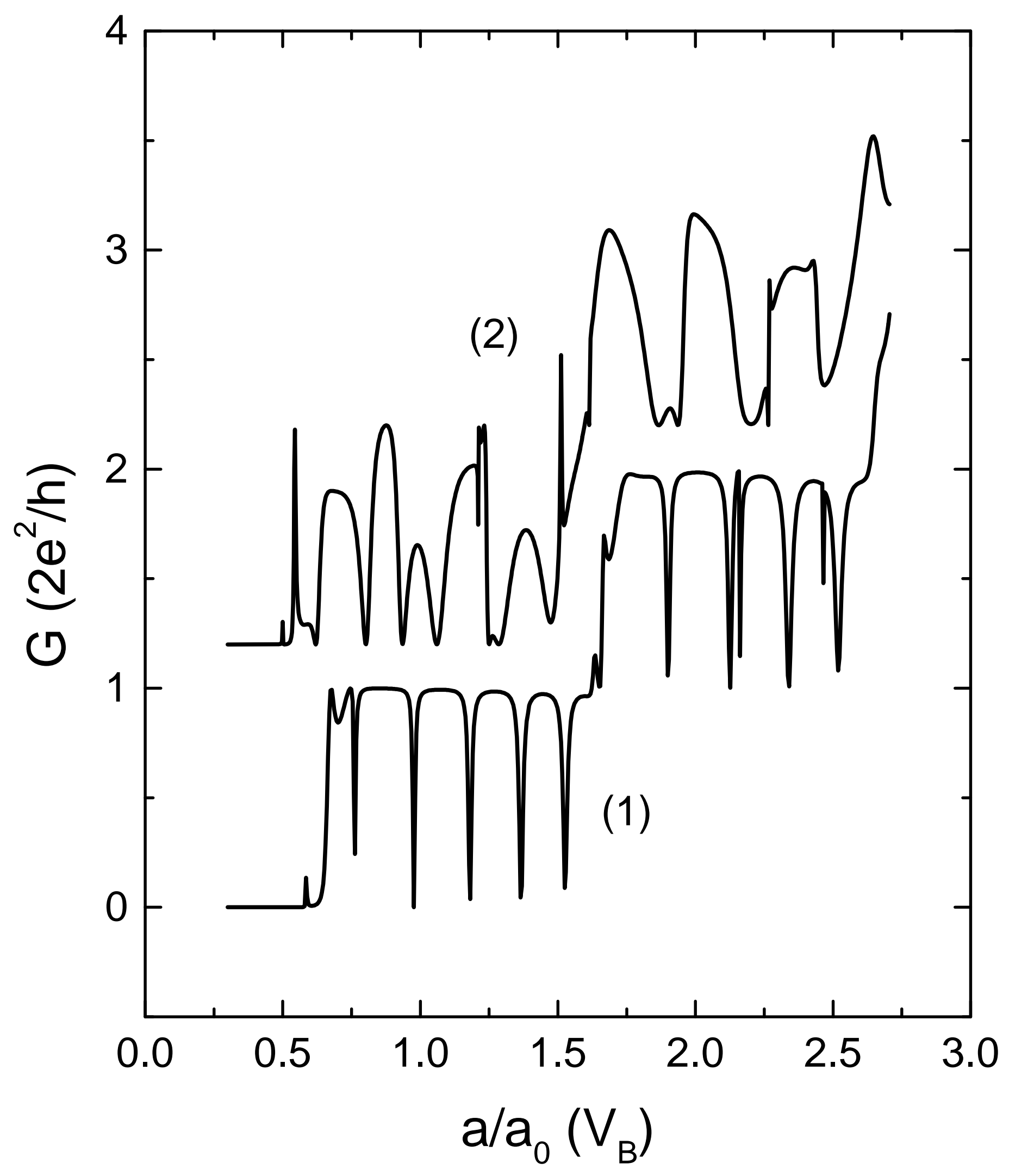

Fig. 10. P. Debray et al. 


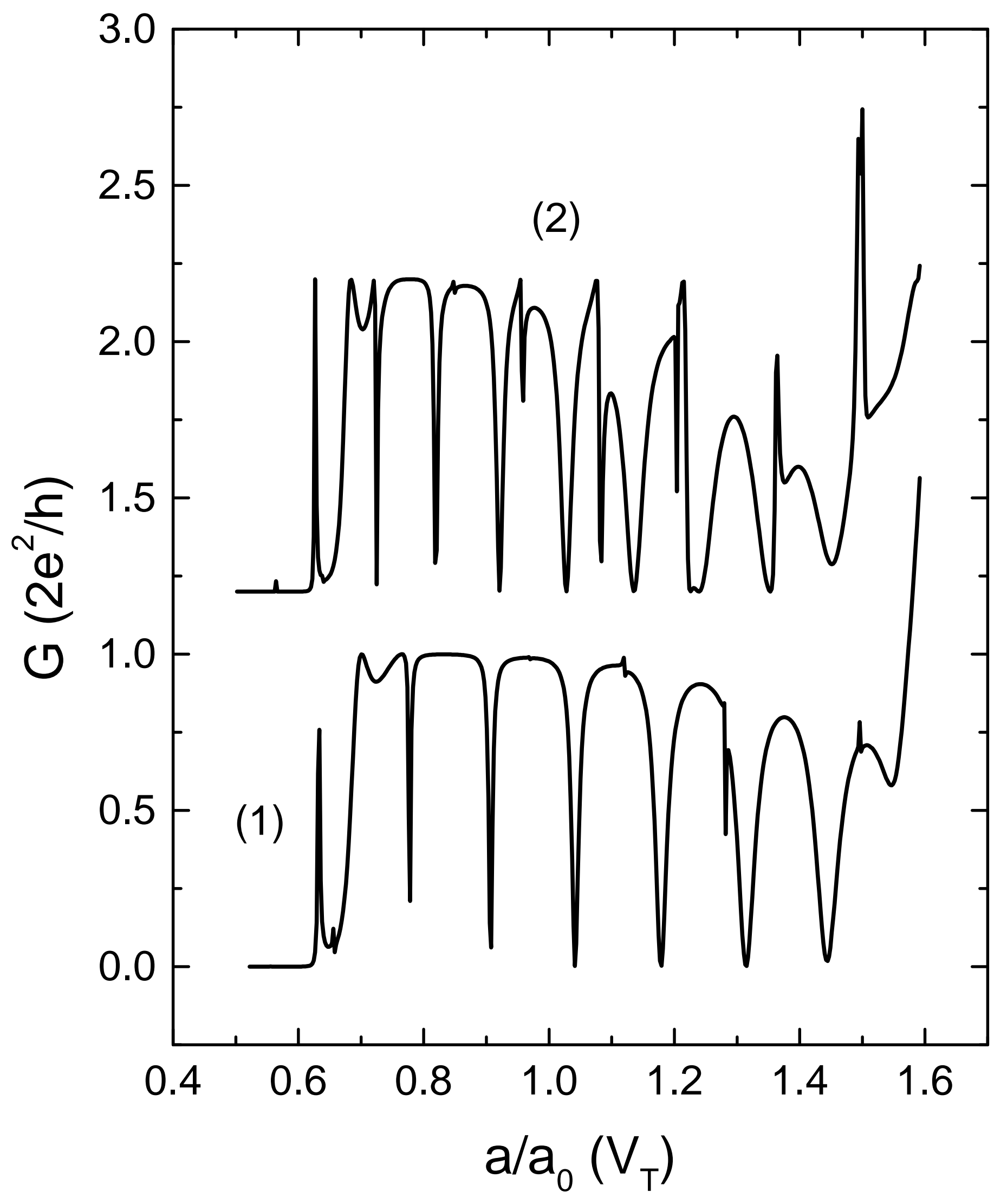

Fig. 11. P. Debray et al. 